Revue

Revue de l'histoire des religions

de Ihistoire des religions

Alexis PINCHARD, Les langues de sagesse dans la Grèce et l'Inde anciennes

Genève, Droz, 2009, X-637 p.

Guillaume Ducœur

\title{
OpenEdition
}

Journals

Édition électronique

URL : http://journals.openedition.org/rhr/7797

DOI : 10.4000/rhr.7797

ISSN : 2105-2573

Éditeur

Armand Colin

Édition imprimée

Date de publication : 1 septembre 2011

Pagination : 434-439

ISBN : 978-2200-92721-9

ISSN : 0035-1423

Référence électronique

Guillaume Ducœur, "Alexis PInCHARD, Les langues de sagesse dans la Grèce et l'Inde anciennes », Revue de I'histoire des religions [En ligne], 3 | 2011, mis en ligne le 12 décembre 2011, consulté le 21 septembre 2020. URL : http://journals.openedition.org/rhr/7797 ; DOI : https://doi.org/10.4000/rhr.7797

Ce document a été généré automatiquement le 21 septembre 2020

Tous droits réservés 


\section{Alexis PINCHARD, Les langues de sagesse dans la Grèce et l'Inde anciennes}

Genève, Droz, 2009, X-637 p.

Guillaume Ducœur

\section{RÉFÉRENCE}

Alexis Pinchard, Les langues de sagesse dans la Grèce et l'Inde anciennes, Genève, Droz, 2009, X-637 p., $23 \mathrm{~cm}$ ("École pratique des hautes études. Sciences historiques et philologiques. III, Hautes études du monde gréco-romain », 43)

Dans cet ouvrage, issu d'une thèse de doctorat soutenue à l'École Pratique des Hautes Études (section des sciences historiques et philologiques), Alexis Pinchard (AP) propose une révision de l'histoire des fondements de la philosophie platonicienne, plus particulièrement de la théorie des Idées, afin de redéfinir la notion même de oopí $\alpha$. Après avoir énoncé dans l'introduction les présupposés requis, à savoir les parentés linguistique, mythologique et poétique indo-européennes (dorénavant i-e) entre la Grèce et l'Inde anciennes ainsi que l'appel aux sources védiques dans l'optique d'une démarche comparative heuristique, l'auteur articule sa démonstration en trois parties.

Dans la première («Une méthode nouvelle pour un problème ancien »), AP réexamine la notion aristotélicienne de sagesse ( $\sigma \circ \varphi \hat{i} \alpha)$ en tant que science et montre que celle-ci n'avait pas encore, au temps des présocratiques et de Socrate lui-même, le sens épistémique qu'Aristote lui prêta mais qu'elle relevait plus de l'excellence verbale. Afin de mieux saisir les causes historiques à l'origine de sa "désagrégation progressive " (90) qui a abouti à sa polysémie, l'auteur considère que la méthode éprouvée dans les domaines de la grammaire et de la mythologie comparées i-e peut également être appliquée à la philosophie dès lors que la sagesse repose avant tout sur un héritage métalinguistique commun à la Grèce et à l'Inde dont la langue des dieux - et par extension la langue de sagesse ou des sages - en opposition à la langue du commun des mortels, demeure le substrat i-e le plus évident à repérer et à restituer. 
3 La deuxième partie ("La langue des dieux: une pratique sapientiale exemplaire »), cœur de la démonstration, porte sur l'essence des noms dans la langue des dieux et plus particulièrement sur les «noms secrets » des dieux rgvédiques et la reconstitution d'un formulaire i-e de double nomination. Le repérage du syntagme "sur le dos du ciel»

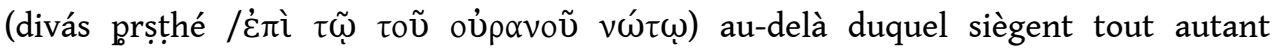
l'hypostase hénothéique suprême des dieux védiques voire des dieux grecs - dans le cas de Dionysos - que le Veda lui-même, suggère une possible origine i-e du concept platonicien du monde intelligible des Idées au-delà duquel siège l'essence des choses vers laquelle tendent les âmes du monde sensible. Platon apparaîtrait dès lors comme l'héritier de la sagesse des poètes i-e, fondée sur la langue des dieux, qui aurait été conservée et transmise, encore en partie, à travers les rites initiatiques des Mystères d'Eleusis.

4 C'est dans la troisième et dernière partie de l'ouvrage ("Origine, signification et fonction des mystères ») que l'auteur s'interroge sur les liens possibles entre, d'une part, les initiations orphico-éleusiennes et, d'autre part, la théorie de la réminiscence platonicienne exposée en un langage métaphorique qui n'est pas sans rappeler les phases du rituel initiatique mystérique lui-même. Pour parvenir à démontrer que les Mystères relèvent d'une tradition sapientiale structurée selon l'idéologie i-e, AP compare en premier lieu les natures et fonctions communes des couples DionysosPerséphone et Agni-Ușas puis retrouve dans la double nomination Sémélè/Thyonè une continuité de la langue des dieux des poètes i-e. Dès lors, l'héritage de cette poétique i-e immortalisante et les correspondances entre le voyage des Pères indo-iraniens jusqu'au "Siège de l'Ordre cosmique " et les étapes initiatiques des Mystères grecs ouvrant l'initié à la connaissance de sa vraie nature divine permettent à l'auteur d'affirmer que la sagesse platonicienne et à sa suite la philosophie grecque s'enracinent dans une telle représentation de la délivrance de l'âme et réactualisent la vision de la nature naturante des poètes inspirés i-e.

5 Cette thèse novatrice, exposée dans un langage conceptuel agréable à lire et dont on suit les étapes successives du raisonnement au fur et à mesure de la lecture des cinq cent quatre-vingt-quinze pages, a le mérite d'essayer de réconcilier les différentes traditions grecques de sagesse ou, selon son auteur, de sauver l'unité de la sagesse platonicienne. Néanmoins, derrière la reconstitution de cette généalogie de la pensée grecque - de la langue des dieux des poètes i-e à la théorie des Idées de Platon en passant par les rites mystériques orphico-éleusiens - on découvre un certain nombre de postures, tant en ce qui concerne la méthode comparative dans le domaine des études i-e que l'approche historico-critique et philologique des sources textuelles, de leur traduction et de leur interprétation, qui nuisent à la démonstration et qui contribuent à en faire plus une herméneutique personnelle qu'une thèse scientifique imparable.

6 Tout au long de son ouvrage, l'auteur est tiraillé entre une démarche comparative heuristique $(7,126)$ - étude de la pensée grecque et de la pensée védique mises en regard - qu'il qualifie parfois de méthode neutre (454) et une étude comparée des sources textuelles grecques et védiques visant à restituer un fonds de sagesse commun i-e. Or, si la première peut être acceptable afin de permettre une nouvelle approche de l'histoire de la philosophie grecque, la seconde demeure plus problématique. Par ses contacts méditerranéens (brève allusion à ce problème seulement dans la conclusion, 591), la Grèce ancienne, en effet, se prête moins bien au comparatisme que les autres 
sphères culturelles de langue i-e. De ce fait, les réductions opérées par l'auteur aboutissent souvent bien plus à une mise en évidence d'une typologie de la sagesse gréco-indienne qu'à une réelle reconstitution i-e. Si, dans l'introduction (4), AP annonce que cette étude sur la бopía grecque se doit de prendre en considération tout autant les sources védiques qu'avestiques, latines et eddiques, le lecteur se rendra rapidement compte que le comparatisme porte avant tout sur les seuls mondes grec et indien. Tout au plus, comptons-nous quelques références, en notes de bas de page, à l'Avesta $(154,155,332)$ - le terme indo-iranien n'apparaissant qu'à la page 547 - à l'Edda (126) et aux textes latins $(486,547,562,568)$. Dans le domaine romain, l'auteur préfère faire appel aux ouvrages de G. Dumézil $(94,98,459,486,562,560)$. Ainsi, loin de faire une étude comparative i-e en tant que telle, de simples analogies gréco-indiennes suffisent, selon lui, à la restitution d'un fonds commun i-e (101, 109, 124, 197, 383, 454, $457,517,533,544,588)$. Cette démarche réductrice est la conséquence d'une visée ambitieuse cherchant à remonter aux origines d'un phénomène, en l'occurrence aux origines non seulement de la sagesse grecque mais encore d'une sagesse i-e $(101,127$, $142,168,303,305,384,455)$, et à énoncer une théorie générale « des lois sapientiales » (9).

7 Le traitement des sources reste le domaine le plus exposé aux réserves. Leur étude demeure plus philosophique et herméneutique qu'historique et philologique. Dans l'introduction, AP prévient son lecteur qu'il ne portera son attention que sur les textes "sans dépendre des aléas de l'archéologie de terrain » (9). Au sujet des rites orphicoéleusiens, il prend toutefois à témoin un bas-relief (404) ou fait une libre interprétation iconologique des peintures murales de la Villa des Mystères de Pompéi (460-461). Délaissant la méthode historico-critique, l'auteur considère l'ensemble de ses sources d'un point de vue synchronique. Les citations sont souvent décontextualisées et semblent n'avoir plus aucune histoire rédactionnelle. Afin d'étayer sa démonstration, l'auteur passe indifféremment $\mathrm{du}_{\mathrm{o}} \mathrm{Rg}$ Veda, aux Brāhmaṇa, aux Upanișad voire même au Mahābhārata, à la Bhagavad Gìtā ou au Rāmāyaṇa. Il en est de même pour les sources gréco-latines s'étalant du viII ${ }^{e}$ siècle av. J.-C. au ve siècle ap. J.-C. Plus de mille ans d'histoire textuelle sans histoire. Le lecteur rencontrera parfois quelques précisions historiques $(303,326,380,384,420,514)$, rarement l'étude de la visée rédactionnelle d'un écrivain antique (453) et constatera, de ce fait, des contradictions dans la pensée de l'auteur $(154 / 342 ; 225 / 547 ; 341 / 347)$.

8 Si ce dernier déclare par ailleurs qu'il donnera «toujours en note le texte original » (p. ix) - ce qui n'est assurément pas le cas tout au long de l'ouvrage (106, 113, 123, 124, 162, 183...) - afin que «le lecteur puisse juger par lui-même de ses choix herméneutiques » (p. ix), ses traductions semblent souvent arbitraires. « Personnelles » ou «modifiées", selon les indications de l'auteur, elles font sens pour la théorie démontrée mais n'en ont plus guère une fois replacées dans leurs contextes proches ou lointains car le choix opéré fait fi de toute critique interne. Ces traductions trahissent souvent la portée sémantique si spécifique des divers termes comparés issus de courants de pensée hétérogènes. En outre, certains passages n'ont pas été traduits comme, par exemple, «paulkaso'paulkasah śramaṇo'śramaṇah » en BĀU 4.3.22 (123) dont le texte original n'est d'ailleurs pas donné. Parfois, la traduction personnelle ne suit pas le texte sanskrit (351). Pour la traduction de Yašt 10.108 (155), seule la référence textuelle est mentionnée, non le texte original, ni le nom du traducteur. La signification de certains substantifs sanskrits ne correspond pas exactement à ce à quoi 
elle se rapporte (par exemple, «afin de procurer l'empire universel au sacrifiant (cakravartin) », 357).

9 Il apparaît qu'AP ne s'est pas donné la peine de faire une véritable étude philologique des termes techniques qu'il a repérés dans les sources sanskrites afin de démontrer solidement sa thèse. Nous ne prendrons ici que quelques exemples. Des pages 98 à 101, il reprend une des esquisses duméziliennes portant sur la trifonctionnalité i-e de la vāc tel que semble en témoigner.RV 10.125.4-6. Sans être critique envers l'étude rapide qu'en avait faite $\mathrm{G}$. Dumézil dans ses esquisses, et qui n'était pour lui qu'un repérage à approfondir et à confirmer, AP en déduit que «les interactions entre les diverses modalités i-e de la parole étant précisées, nous pouvons désormais déterminer en quoi consiste intimement la première d'entre elles, et découvrir qu'elle était déjà définie à l'origine » (101). Or, si G. Dumézil y voyait un héritage de la trifonctionnalité i-e, AP en conclut qu'il s'agit précisément "des modalités indo-européennes de la parole ». Par ailleurs, la théorie d'une bipolarité descendante et ascendante de la vāc due aux seuls -ríși et brahmán laisse de côté sumedhás, terme tout aussi essentiel dans la strophe Orgvédique ( tám brahm'ānam tám oŕṣim tám sumedh'ām»). L'analyse de la vingtaine d'occurrences 。rgvédiques de ce terme, si particulier dans l'hymnaire (notamment $\mathrm{RV}$ 1.185.10 et 3.38.1), aurait certainement permis, d'une part, de compléter l'analyse de G. Dumézil, qui ne l'avait guère lui-même pris en considération, et, d'autre part, de dégager toute la spécificité de ce substantif. La traduction par " sage » est loin de rendre la portée sémantique de ce terme qui, correctement analysé, aurait grandement enrichi l'étude de la figure du poète orgvédique, sa fonction religieuse et son rapport à la vāc.

De même, la traduction et l'interprétation du second hémistiche de Bhagavad Gïtā 15.18 (ato'smi loke vede en pratitah puruṣottamah, «Je suis connu tant dans [la langue] profane que dans le Veda en tant que Purușa suprême », 224), reste très personnelle et ne prend en compte ni la critique interne de l'œuvre ni les contextes historique, religieux et doctrinal qui permettent de mieux saisir le sens de cette affirmation krșnaiite. Par ailleurs, AP passe d'une restitution sémantique «dans [la langue] profane " à une traduction dorénavant acquise mais pourtant fautive «dans la langue profane " (325). À la dichotomie entre langue des dieux et langue des mortels, entre Veda et loka dans ce passage de la Bhagavad Gitā, il conviendrait plutôt de s'interroger sur la relecture korșnaiite de la Śruti. L'analyse sémantique du védique jihv' ā (177) n'est guère plus convaincante, tout comme l'idée de "noms secrets" atharvaniques rapprochés des noms inétymologisables des Papyri graecae magicae (188).

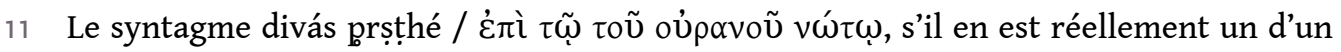
point de vue de la poétique i-e - ce qui resterait encore à démontrer - aurait mérité une étude plus approfondie d'autant qu'il est l'un des arguments forts de l'auteur. Là encore, AP ne fait que mettre en parallèle des tournures stylistiques sans pour autant les étudier (divás prșțhé en $。$ RV $1.115 .3 ; 1.166 .5 ; 3.2 .12 ; 9.36 .6 ; 9.66 .5 ; 9.69 .5 ; 9.83 .2$ mais également $n^{\prime}$ àkasya prșthé en 。RV $1.125 .5 ; 3.2 .12$ ne sont pas mentionnés) dans leurs contextes cosmologique et rituel, ni prendre en considération, comme il le fait parfois, leur réemploi postérieur (par exemple Mahābhārata [éd. Poona] 12.12.5; $12.12 .36 ; 13.105 .40)$. À défaut d'une argumentation fondée sur une analyse philologique minutieuse, il ne peut plus que s'étonner des analogies repérées: "N'est-il pas frappant, en effet, que l'expression 'sur le dos du ciel', propre à la liturgie somique, revienne spécialement dans le mythe psychique de Phèdre qui expose la transcendance 
de l'essence à l'égard du sensible sans se cacher d'épouser le cadre de l'initiation éleusienne?»(377).

L'étude comparée des natures et fonctions communes des couples Dionysos-Perséphone et Agni-Ușas est également loin d'être concluante et ce d'autant moins que la plupart des arguments sont affaiblis constamment par des incertitudes dues en grande partie au comparatisme analogique dans lequel s'est enfermé l'auteur (« Peut-être devonsnous ainsi interpréter... », 105 ; "Admettons, malgré tout, que la différence... », 185 ; «Certes la citation ne mentionne pas explicitement... », 417 ; « Certes le propos est très ambigu... », 422 ; «... des analogies troublantes... l'aveu répété et raisonné, quoique discret... ", 423 ; "Certes la déesse grecque de l'aurore, Eôs, ne porte pas ce titre, mais... ", 477 ; " On objectera peut-être que... ", 514, 533, 536, 560, 577). En matière d'histoire comparée des religions, de mythologie comparée ou de philosophie comparée, le comparatisme différentiel demeure fondamental or ce dernier n'a pas été mis à profit.

Dans la bibliographie, les classements des sources sanskrites « par ordre de sacralité et de dépendance " ou "par ordre chronologique supposé " sont originaux mais non nécessaires, d'autant moins que l'étude historico-critique de ces mêmes sources n'a guère été la priorité de l'auteur. On s'étonnera, tout de même, de voir J. Muir en tête de la Smorti (599) et de l'absence d'un certain nombre de sources, notamment avestiques, eddiques et surtout gréco-latines, pourtant citées dans l'ouvrage (Andocide, 565; Euripide, 474 et 565 ; Lucrèce 555 ; Macrobe, 470 ; Maxime de Tyr, 541 ; Ovide, 476 ; Philon, 471 ; Plutarque, 476 ; Pseudo-Platon, 470 ; Virgile, 568).

L'index n'est pas aisément exploitable car l'auteur n'a ni donné les références complètes des citations tant grecques que sanskrites - les strophes des hymnes irgvédiques, par exemple, ne sont pas mentionnées - ni reporté la liste exhaustive des sources citées. Ainsi, l'absence de toute référence à la Bhagavad Gītā (224 et 325), à Andocide (565), Aristophane (471), Diogène Laërce (551), Hippolyte (561), Lucrèce (555), Macrobe (470 et 486), Maxime de Tyr (541), Nonnos (463), Ovide (476), Philon (471), Porphyre (484), Pseudo-Platon (470) et Virgile (568) est notoire.

La thèse soutenue par AP n'emporte pas la conviction, non que la problématique ne soit pas pertinente, mais parce que toute l'étude repose sur un comparatisme analogique qui fausse continuellement l'analyse des sources et leur interprétation. Pris entre comparatisme heuristique (Grèce/Inde) et reconstitution d'une sagesse commune i-e dont l'héritage serait encore inscrit dans l'histoire de la pensée présocratique, platonicienne et védique et dans l'histoire rédactionnelle des sources textuelles, AP ne nous semble pas parvenir à démontrer scientifiquement son intuition. Souhaitons que l'auteur puisse retravailler son "mirage » i-e en recourant davantage aux méthodes éprouvées propres aux historiens et aux philologues. 


\section{AUTEUR}

GUILLAUME DUCCEUR

Université de Strasbourg 